\title{
Suplementação de iodo na pré-concepção, gravidez e amamentação: a recomendação e a Medicina baseada na inferência
}

Filipa Silva*, José Agostinho Santos**

\begin{abstract}
RESUMO
Em Agosto de 2013, a Direcção-Geral da Saúde publica a orientação técnica 011/2013, recomendando a suplementação de iodo em mulheres na pré-concepção, gravidez e amamentação, sob a forma de iodeto de potássio (150-200 $\mu \mathrm{g} / \mathrm{dia}$ ). Esta orientação vai ao encontro das recomendações da Organização Mundial de Saúde e da American Thyroid Association e dos resultados de estudos observacionais. Um desses estudos foi realizado em Portugal e incluiu 3.631 grávidas portuguesas, tendo revelado uma baixa iodúria, principalmente entre as açorianas, madeirenses e residentes no interior do continente. Pensa-se que baixa iodúria significa baixa iodemia, algo que por sua vez conduz a baixos níveis de hormonas tiroideias e consequentemente a alterações neurocognitivas. No entanto, questiona-se se a suplementação generalizada com iodo nestas mulheres terá impacto na diminuição da morbilidade do feto e da criança. $O$ presente artigo reflecte sobre a orientação técnica à luz da Medicina baseada na evidência e orientada para o paciente.
\end{abstract}

Palavras-chave: lodo; Suplementos Dietéticos; Gravidez.

\section{A RECOMENDAÇÃO DA DIRECÇÃO-GERAL DE SAÚDE}

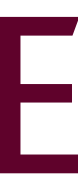

m Agosto de 2013, a Direcção-Geral da Saúde (DGS) publicou a orientação técnica 011/2013, recomendando a suplementação de iodo às mulheres em pré-concepção, grávidas e em amamentação exclusiva. Os médicos são instados a recomendar iodeto de potássio, na dosagem de 150-200 $\mu \mathrm{g} /$ dia, e a aconselhar uma alimentação variada que inclua fontes de iodo (particularmente pescado, leguminosas, produtos hortícolas e produtos lácteos). ${ }^{1}$ Esta recomendação teve destaque nos media e surpreendeu a comunidade médica portuguesa. ${ }^{2}$

Esta orientação é justificada pela importância que o iodo assume no processo de desenvolvimento e matu-

*Interna de Formação Específica de Medicina Geral e Familiar, USF Dunas, ULS Matosinhos.

**Assistente de Medicina Geral e Familiar, USF Dunas, ULS Matosinhos. ração do sistema nervoso central do feto e futuro paciente pediátrico. ${ }^{3,4} \mathrm{~A}$ orientação destaca o papel do iodo mediante a sua integração na composição das hormonas tiroideias que, por sua vez, têm um papel activo na regulação do metabolismo celular e estimulação do crescimento e desenvolvimento orgânico. ${ }^{1,3}$ Foram já observadas as funcionalidades destas hormonas em diversos estudos. Sabe-se que, num nível neurocelular, as hormonas tiroideias estão envolvidas na multiplicação, migração, maturação e organização neuronal. ${ }^{4}$ A literatura sugere que esta sucessão de processos se traduz numa optimização da expressão das potencialidades do sistema nervoso, quer a nível cognitivo quer a nível de outras funções cerebrais. ${ }^{4,5}$

O feto apenas produz hormonas tiroideias na segunda metade da gestação. É portanto totalmente dependente dos níveis hormonais maternos durante os primeiros meses e da concentração de iodo circulante 
na mãe durante toda a gravidez, pois este é necessário numa primeira fase para a síntese hormonal materna e posteriormente para a produção de hormonas tiroideias pelo próprio feto. ${ }^{1,45}$ Desta cadeia de dependências sucessivas, sobressai a forte dependência do feto em relação à mãe. Deste ponto também se resgatam na orientação técnica os motivos para uma necessidade aumentada de iodo durante a gestação: existe um aumento das necessidades de tiroxina (T4) para manter o metabolismo normal da grávida e para estimular o metabolismo fetal, ao mesmo tempo que se eleva a depuração renal na grávida. ${ }^{1,6}$ Já os lactentes sob aleitamento materno exclusivo, embora já sintetizadores das suas próprias hormonas, têm o seu aporte de iodo através do leite materno em estreito compromisso com a ingestão alimentar da mãe, carimbando a forte relação de dependência já iniciada durante a gravidez. Os lactentes sob aleitamento artificial adquirem o seu iodo através das fórmulas lácteas já fortificadas. ${ }^{1,7}$

Estando os valores circulantes deste oligoelemento em próximo acordo com a quantidade de hormonas tiroideias produzidas e, por sua vez, em íntima relação com a sua ingestão alimentar, diversos autores têm salientado, desde há alguns anos, a necessidade de reforçar a ingestão de iodo durante o período de pré-concepção, gravidez e aleitamento. ${ }^{4}$ Destacam igualmente os riscos subjacentes a um baixo aporte alimentar de iodo, que se relaciona tanto com o diminuto volume ingerido dos produtos mais ricos neste elemento como com um possível reduzido teor mineral na água e nos solos de onde provêm, directa ou indirectamente, esses mesmos produtos. ${ }^{1}$

As denominadas perturbações por défice de iodo têm como consequência o hipotiroidismo e um inadequado desenvolvimento cognitivo e/ou comportamental. ${ }^{1,3,4} \mathrm{~A}$ orientação da DGS destaca que o défice de iodo é uma das causas evitáveis de alterações do desenvolvimento ou de doenças mentais e salienta, em particular, os resultados de três estudos observacionais realizados em Portugal que convergem na conclusão de que a maioria das grávidas portuguesas apresentam um aporte alimentar de iodo que fica abaixo das necessidades materno-fetais., ${ }^{1,8,9,10,11}$ Poderá ficar subentendido para o leitor que consulta a orientação vigente que foram estes resultados os fortes motivadores da elaboração desta recente recomendação de suplemen- tação farmacológica de iodo. É, por isso, de crucial importância analisar estes estudos, já que constituem a premissa-base (real défice de iodo) para a cadeia lógica de deduções que advém da mesma.

\section{DÉFICE DE IODO NAS GRÁVIDAS PORTUGUESAS: OS RESULTADOS CENTRAIS}

Em 2010 e 2012 foram publicados os resultados do maior estudo transversal em torno dos níveis de iodo nas grávidas portuguesas, envolvendo um total de 3.631 mulheres seguidas nos cuidados de saúde secundários. ${ }^{8,9}$ Foi usada a concentração urinária de iodo (iodúria) como indicador da sua ingestão alimentar, uma vez que aproximadamente $90 \%$ do iodo consumido é excretado na urina. ${ }^{1,3}$ Estes resultados revelaram níveis inferiores aos considerados satisfatórios pela Organização Mundial de Saúde (OMS): ${ }^{12}$ somente $17 \%$ das grávidas de Portugal Continental apresentam níveis de iodúria satisfatórios e as mulheres do interior têm um défice mais acentuado do que as do litoral. Entre as grávidas dos arquipélagos existe um défice ainda mais notório: $92 \%$ das madeirenses apresentam baixa iodúria, subindo para $99 \%$ entre as açorianas. ${ }^{8,9}$ Globalmente, a média de iodúria situou-se em 82,5 $\mu \mathrm{g} / \mathrm{dL}$, valor que corresponde a uma região de défice ligeiro. ${ }^{8,9,13}$

Outros dois estudos realizados no Minho mostram que mesmo as mulheres em idade fértil (e, portanto, potencialmente em pré-concepção) apresentam valores deficitários de iodúria e os valores sanguíneos de hormonas tiroideias entre a maioria das grávidas eram insuficientes, atendendo aos padrões estabelecidos pela OMS..$^{10,11}$

Estes estudos são, por si só, muito relevantes, uma vez que envolvem pacientes portuguesas. Na literatura médica não são comuns os estudos que forneçam dados nacionais.

Os resultados destes estudos observacionais vieram alertar os profissionais de saúde para as aparentes deficiências do aporte de iodo entre as mulheres habitantes em Portugal, desencadeando uma corrente de preocupações em torno dos riscos para os fetos e futuras crianças portuguesas: uma baixa iodúria significaria um baixo aporte nutricional materno e, eventualmente, levaria a perturbações do desenvolvimento e crescimento cognitivo-comportamental para os seus filhos. A suplementação de iodo nas mulheres portuguesas seria assim baseada numa premissa que parece 
lógica: a suplementação elevaria os níveis de iodemia e evitaria toda a cadeia de eventos negativos já descrita.

De facto, desde há alguns anos que a literatura médica é pródiga em artigos em torno deste tema relativamente polémico, não havendo um consenso quanto à necessidade de suplementação de iodo. Existem estudos em que se conclui que a suplementação em regiões com défice ligeiro a moderado não terá impacto na redução da morbilidade. ${ }^{14,15} \mathrm{~A}$ OMS recomenda esta suplementação, mas sustentando-se em dados de estudos observacionais. ${ }^{12}$ Já outras associações científicas, como a American College of Obstetricians and Gynecologists e a National Institute for Health and Clinical Excellence, adoptam posições neutras, não se pronunciando sobre esta suplementação nas suas normas de orientação clínica. ${ }^{16,17}$ Somente a American Thyroid Association recomenda a suplementação geral. ${ }^{18} \mathrm{~A}$ ausência de consenso pode dever-se ao facto de a evidência não ser suficientemente sólida quando se estuda a suplementação com iodo quanto a resultados orientados para a mãe ou para o feto (como, por exemplo, a redução estatisticamente significativa de eventos adversos como hipotiroidismo, atraso de crescimento ou diminuição da performance intelectual, entre outros) em vez de orientados para a doença.

Estes resultados orientados para a mãe ou para o feto inserem-se no conceito de evidence that matters. Os estudos observacionais documentam níveis de iodo nas populações estudadas inferiores aos recomendados e sabe-se que as hormonas tiroideias têm um papel importante no desenvolvimento do sistema nervoso central do feto/criança. Já a eficácia e a segurança da suplementação generalizada de iodo a todas as mulheres que pretendem engravidar, grávidas e em período de amamentação exclusiva na redução de morbilidade tiroideia e neurocognitiva dos seus filhos, não é fortemente sustentada pela evidência científica actual, como veremos adiante. Esta forma de estabelecer recomendações com base em deduções a partir de factos conhecidos será denominada no presente artigo como Medicina baseada na inferência.

\section{MEDICINA BASEADA NA INFERÊNCIA}

O artigo de revisão de Zimmermann e Delange constitui uma das referências bibliográficas da orientação
011/2013 da DGS e, apesar de ter sido publicado em 2004, reúne os principais estudos realizados em torno da suplementação de iodo, na sua maioria ensaios clínicos aleatorizados e controlados. ${ }^{19}$ Esta tipologia de estudo compara resultados entre grupos relativamente semelhantes e em que a única diferença existente é a intervenção aplicada (neste caso, a administração oral de iodo). ${ }^{20}$

Zimmermann e Delange debruçaram-se sobre seis ensaios clínicos, envolvendo um total de 450 grávidas: todos compararam os níveis de iodúria entre as suplementadas e as não-suplementadas. Foram também avaliados em alguns destes estudos outros aspectos como os valores séricos de TSH, T4 e tiroglobulina e o volume da glândula tiroidea (na mãe ou no filho). Numa apresentação geral dos resultados, todos documentaram um aumento estatisticamente significativo dos níveis de iodúria materna com a suplementação de iodo, sem efeitos laterais significativos (o que salienta a sua aparente segurança). Em três dos seis ensaios, a suplementação levou a um menor aumento compensatório das glândulas tiroideias das grávidas intervencionadas, comparativamente às não suplementadas. Quanto aos restantes outcomes avaliados, a evidência é díspar, não permitindo estabelecer claras relações entre a suplementação e possíveis benefícios. ${ }^{19}$

No entanto, embora com metodologia adequada a determinar a eficácia de uma intervenção, o que emerge de imediato neste conjunto de evidência científica é a natureza dos aspectos medidos como eficácia da suplementação: todos eles orientados para surrogate endpoints, ou seja, passos intermédios na linha que une a suplementação de iodo na mãe e a patologia tiroideia e/ ou neurológica no filho. De facto, numa pesquisa sistemática entre as principais fontes de artigos não são encontrados ensaios clínicos aleatorizados e controlados que comparem suplementação generalizada de iodo a mulheres de uma população geral de região com défice ligeiro de iodo versus não-suplementação quanto a patient-oriented-outcomes, ou seja, quanto ao impacto dessa suplementação na redução da morbimortalidade dos seus filhos (isto ao contrário do que é encontrado, por exemplo, para a suplementação materna de ácido fólico). ${ }^{21}$

Os médicos são portanto conduzidos a praticar uma Medicina baseada na inferência, acreditando que a su- 
plementação terá um efeito benéfico na morbimortalidade por se ter provado que há benefícios em disease-oriented outcomes (parâmetros que estão eventualmente relacionados com a patologia).

Existem dois estudos orientados para o paciente (e não para a doença), mas um é um ensaio clínico não aleatorizado e outro é um estudo observacional (coorte histórica). Estes desenhos não produzem evidência suficientemente robusta para recomendar uma intervenção farmacológica pois, não sendo garantida a semelhança entre os grupos de mulheres comparados (incluindo o afastamento de vieses), não se pode concluir que as diferenças encontradas se devem à intervenção.

Em 2011, é publicado um ensaio clínico não aleatorizado envolvendo 133 grávidas espanholas em que todas foram suplementadas com $300 \mu$ g de iodeto de potássio. Os seus filhos foram posteriormente avaliados sob o ponto de vista de desempenho neuropsicológico, usando instrumentos como Mental Development Scale e Psychomotor Development Index, e comparados posteriormente com os filhos de um grupo de mulheres que não foram suplementadas. Este estudo revelou diferenças estatisticamente significativas entre os dois grupos, realçando melhores resultados entre os filhos de mulheres suplementadas. ${ }^{22,23}$ Neste estudo espanhol, o controlo dos potenciais factores confundidores não foi óptimo, desconhecendo-se igualmente se os benefícios do tratamento se sobrepuseram aos eventuais riscos/custos.

Em 2013, é publicado no Reino Unido um estudo observacional em que foi avaliada a performance cognitiva dos filhos de 1.040 grávidas que foram retrospectivamente divididas em duas categorias: as que no primeiro trimestre tinham iodúria $<150 \mu \mathrm{g} / \mathrm{mL}$ (carenciadas em iodo) e aquelas com iodúria $>150 \mu \mathrm{g} / \mathrm{mL}$ (não carenciadas). Aos seus filhos foi aplicado um teste de QI aos 8 anos de idade. Os autores tentaram identificar factores intervenientes no desenvolvimento neurológico que poderiam ser confundidores e fornecedores de vieses. Isolaram 21 desses factores e ajustaram as comparações de acordo com eliminação de confundidores. Os resultados revelaram que os filhos de mulheres carenciadas apresentavam uma redução estatisticamente significativa nos valores de QI comparativamente aos filhos de mulheres não-carenciadas no pri- meiro trimestre de gravidez. ${ }^{24}$ É portanto sugerida uma associação entre os valores de iodúria e a morbilidade pediátrica, mas não estabelece, na realidade, uma associação que seria relevante para a discussão: entre a suplementação de iodo e a morbilidade. Finalmente, é discutível se os 21 factores isolados como confundidores neste estudo são, na realidade, a totalidade dos factores intervenientes.

Além do já referido, são variados os factores que modificam a farmacodinâmica e segurança dos compostos disponíveis contendo iodeto de potássio, ${ }^{19}$ que deverão ser estudados aquando do registo de benefícios orientados para o paciente.

Relativamente aos potenciais benefícios da suplementação no período de pré-concepção e amamentação, os estudos são ainda mais escassos.

Um outro aspecto importante mas envolto de pouca ou nenhuma evidência prende-se com o potencial efeito tóxico do iodo tanto ao nível da saúde materna como no próprio desenvolvimento fetal. É já reconhecido que uma dieta excessiva em iodo pode aumentar o risco de tiroidite, hipotiroidismo, hipertiroidismo e bócio. ${ }^{25}$ Um estudo observacional documentou disfunção tiroidea em grávidas provindas de regiões de défice ligeiro, ${ }^{26}$ mas não existe evidência robusta publicada acerca dos efeitos tóxicos da suplementação generalizada no que toca a resultados orientados para o paciente. Não há igualmente garantias que a administração de iodo a mulheres sem qualquer défice seja segura.

O mesmo sucede com o perfil de segurança na criança amamentada. Num estudo observacional realizado por Zimmermann et al, foram analisados cerca de 3.319 crianças entre os 6 e os 12 anos. A cada criança foi calculada a excreção urinária de iodo de 24 horas e foi medido o volume da tiróide. Constatou-se apenas um aumento do volume da tiróide para o grupo que detinha o valor mais elevado de excreção urinária $(741 \mu \mathrm{g} / \mathrm{dia}) .^{27}$ Este tipo de trabalho gera evidência científica de robustez muito limitada.

Perpetuando uma Medicina baseada na inferência, haverá ainda um ponto comum aos estudos portugueses, apresentados anteriormente, que permite questionar a extrapolação dos seus dados para a população geral de grávidas: as mulheres incluídas eram vigiadas nos cuidados de saúde secundários, algumas desde $o$ 
primeiro trimestre. Poderá pensar-se, portanto, que eram grávidas de maior risco, pois as grávidas da população geral são seguidas fora do hospital até ao final do terceiro trimestre, excepto se existir risco gestacional. Seria essencial caracterizar e comparar as grávidas vigiadas nos cuidados de saúde primários e as vigiadas nos cuidados de saúde secundários quanto a aspectos que possam influenciar a ingestão, metabolismo e excreção de iodo, tais como a idade, patologias associadas, tipo de alimentação e perfil socioeconómico, entre outros. As diferenças entre a população dos cuidados secundários e a população dos cuidados de saúde primários são, de resto, debatidas em diversas esferas da investigação médica, sendo complexa a aplicação à população geral de dados encontrados na população dos cuidados secundários. ${ }^{28}$

\section{CONCLUSÕES}

Em Agosto de 2013 foi publicada a orientação técnica da DGS que recomenda a suplementação de iodo a todas as mulheres em pré-concepção, gravidez e amamentação, sendo os médicos instados a prescrever um suplemento, para além de aconselharem uma dieta rica neste oligoelemento. É importante que estes profissionais conheçam a escassez de estudos existente no seio da evidência científica actual sobre este tema, essencialmente pela falta de ensaios clínicos aleatorizados e controlados que comprovem que esta suplementação de iodo (quer sob a forma de comprimidos/cápsulas, aqui em causa, quer sob a forma de iodação generalizada do sal) traz, de facto, melhorias nos resultados orientados para os pacientes (mães e filhos) e que é segura. É ainda necessário clarificar o risco/benefício desta suplementação em regiões com diferentes défices de iodo (ligeiro, moderado ou severo) versus um aumento de consumo de alimentos ricos em iodo.

Em conclusão, esta orientação técnica parte da convicção de que, após anos de suplementação de iodo em larga escala, vão reduzir-se significativamente as perturbações do desenvolvimento neurológico e cognitivo entre as crianças, e de que essa redução ultrapassará possíveis riscos tanto para as mães como para os seus filhos. No entanto, neste momento, não há evidência robusta que suporte esta convicção. A evidência científica mais robusta terá, como sempre, a última palavra.

\section{REFERÊNCIAS BIBLIOGRÁFICAS}

1. Direcção-Geral da Saúde. Aporte de iodo em mulheres na preconcecão, gravidez e amamentação. Lisboa: Direcção-Geral da Saúde; 2013.

2. Lusa. Grávidas e mulheres a amamentar aconselhadas a tomar suplemento de iodo. 2013. Disponível em: http://sicnoticias.sapo.pt/pais/ 2013/08/26/gravidas-e-mulheres-a-amamentar-aconselhadas-atomar-suplemento-de-iodo [acedido em 16/09/2013].

3. Pérez-López FR. lodine and thyroid hormones during pregnancy and postpartum. Gynecol Endocrinol 2013 Jul; 23 (7): 414-28.

4. Australian Government. National Health and Medical Research Council. lodine supplementation during pregnancy and lactation: literature review. Disponível em: http://www.nhmrc.gov.au/_files_nhmrc/file/publications/synopses/new45-literature-review.pdf [acedido em 20/09/2013].

5. Santana Lopes M, Jácome de Castro J, Marcelino M, Oliveira MJ, Carrilho F, Limbert E; Grupo de Estudos da tiróide. Iodo e tiróide: o que o clínico deve saber. Acta Med Port 2012 Mai-Jun; 25 (3): 174-8.

6. Brantsaeter AL, Abel MH, Haugen M, Meltzer HM. Risk of suboptimal iodine intake in pregnant Norwegian women. Nutrients 2013 feb 6; 5 (2): $424-40$.

7. Decreto-Lei n 220/99: "Composição de base das fórmulas para lactentes quando reconstituídas de acordo com as instruções do fabricante". Diário da Republica, $1^{\text {a }}$ série A, 16/06/1999.

8. Limbert E, Prazeres S, São Pedro M, Madureira D, Miranda A, Ribeiro M, et al. lodine intake in Portuguese pregnant women: results of a countrywide study. Eur J Endocrinol 2010 Oct; 163 (4): 631-5.

9. Limbert E, Prazeres S, São Pedro M, Madureira D, Miranda A, Ribeiro M, et al. Aporte de iodo nas Regiões Autónomas da Madeira e dos Açores. Rev Port Endocrinol Diabetes Metab 2012 Jul-Dez; 7 (2): 2-7.

10. Costeira MJ, Oliveira P, Ares S, de Escobar GM, Palha JA. lodine status of pregnant women and their progeny in the Minho region of Portugal. Thyroid. 2009 Feb; 19 (2): 157-63.

11. Costeira MJ, Oliveira P, Ares S, de Escobar GM, Palha JA. Parameters of thyroid function throughout and after pregnancy in a iodine-deficient population. Thyroid 2010 Sep; 20 (9): 995-1001.

12. WHO Secretariat, Andersson M, de Benoist B, Delange F, Zupan J. Prevention and control of iodine deficiency in pregnant and lactating women and in children less than 2-years-old: conclusions and recommendations of the Technical Consultation. Public Health Nutr 2007 Dec; 10 (12A): 1606-11.

13. Benoist B, Anderson M, Egli I, Takkouche B, Allen H, editors. Iodine Status Worldwide: WHO Global Database on lodine Deficiency. Geneva: WHO; 2004. Disponível em: http://whqlibdoc.who.int/publications/2004/9241592001.pdf [acedido em 19/11/2013].

14. Zhou SJ, Anderson AJ, Gibson RA, Makrides M. Effect of iodine supplementation in pregnancy on child development and other clinical outcomes: a systematic review of randomized controlled trials. Am J Clin Nutr 2013 Nov; 98 (5): 1241-54.

15. Moral E. Suplementos de yodo en embarazadas sanas. AMF: Actualiz Med Fam 2011 Dic; 7 (11): 647-50.

16. American College of Obstetricians and gynecologists. Subclinical hypothyroidism in pregnancy. ACOG Committee Opinion 2007; 381. Disponível em: http://www.acog.org/ /media/Committee\%200pinions/Committee\%20on\%20Obstetric\%20Practice/co381.pdf?dmc=1 
\&ts=20131209T1032563195 [acedido em 10/11/2013].

17. National Institute for Health and Clinical Excellence. Antenatal Care. NICE Clinical Guideline 62; 2010: 10-2.

18. Public Health Committee of the American Thyroid Association. Iodine supplementation for pregnancy and lactation - United States and Canada: recommendations of the American Thyroid Association. Thyroid 2006 Oct; 16 (10): 949-51.

19. Zimmermann M, Delange F. lodine supplementation of pregnant women in Europe: a review and recommendations. Eur J Clin Nutr 2004 Jul; 58 (7): 979-84.

20. Ebell MH, Siwek J, Weiss BD, Woolf SH, Susman J, Ewigman B, et al. Strength of Recommendation Taxonomy (SORT): a patient-centered approach to grading evidence in the medical literature. Am Fam Physician 2004 Feb; 69 (3): 548-56.

21. De Regil LM, Fernández Gaxiola AC, Dowswell T, Peña Rosas JP. Effects and safety of periconceptional folate supplementation for preventing birth defects. Cochrane Database Syst Rev 2010 Oct 6; 10: CD007950.

22. Velasco I, Carreira M, Santiago P, Muela JA, García-Fuentes E, SánchezMuñoz B, et al. Effect of iodine prophylaxis during pregnancy on neurocognitive development of children during the first two years of life. J Clin Endocrinol Metab 2009 Sep; 94 (9): 3234-41.

23. Yarrington C. Pearce EN. lodine and pregnancy. J Thyroid Res 2011: 934104.

24. Bath SC, Steer CD, Golding J, Emmett P, Rayman MP. Effect of inadequate iodine status in UK pregnant women on cognitive outcomes in their children: results from the Avon Longitudinal Study of Parents and Children (ALSPAC). Lancet 2013 Jul 27; 382 (9889): 331-7.

25. Pennington JA. lodine toxicity. Springfield, VA: National Technical Information Service, US Department of Commerce; 1989.

26. Rebagliato M, Murcia M, Espada M, Alvarez-Pedrerol M, Bolúmar $F$, Vioque J, et al. lodine intake and maternal thyroid function during pregnancy. Epidemiology 2010 Jan; 21 (1): 62-9.

27. Zimmermann MB, Ito Y, Hess SY, Fujieda K, Molinari L. High thyroid volume in children with excess dietary iodine intakes. Am J Clin Nutr 2005 Apr; 81 (4): 840-4.

28. Ruano-Ravina A, Pérez-Ríos M, Barros-Dios JM. Population-based versus hospital-based controls: are they comparable? Gac Sanit 2008 Nov-Dec; 22 (6): 609-13.

\section{CONFLITO DE INTERESSES:}

Os autores declaram não ter conflito de interesses.

\section{ENDEREÇO PARA CORRESPONDÊNCIA}

Filipa Silva

Rua da Cruz, 603, 4455-116 Lavra

E-mail: fi_msilva@hotmail.com

Recebido em 23/09/2013

Aceite para publicação em 24/11/2013

\begin{abstract}
IODINE SUPPLEMENTATION BEFORE AND DURING PREGNANCY AND BREASTFEEDING: RECOMMENDATIONS AND INFERENCE-BASED-MEDICINE

In August 2013, the Portuguese Directorate General of Health published a recommendation for supplementation with potassium iodide (150-200 $\mu \mathrm{g} /$ day) before and during pregnancy and breastfeeding. This recommendation joins to the recommendations of the World Health Organization, the American Thyroid Association, and the results of observational studies. One of these studies was conducted in Portugal with 3631 pregnant women. It found a low level of urinary iodine, especially in pregnant women living in the Azores, Madeira and in the hinterland. It is believed that low urinary iodine levels reflect a low level of serum iodine. This in turn can lead to low levels of thyroid hormones and consequently neurocognitive disorders. The question arises whether iodine supplementation for these women will reduce morbidity in the fetus and the newborn related to low levels of thyroid hormones. We discuss this recommendation in the light of evidence-based and patient-oriented medicine.
\end{abstract}

Keywords: lodine; Dietary Supplements; Pregnancy. 